\title{
Robust Path-Following for UAV Using Pure Pursuit Guidance
}

\author{
Takeshi Yamasaki, Hiroyuki Takano and Yoriaki Baba \\ National Defense Academy \\ Japan
}

\section{Introduction}

This chapter presents a description of a guidance and control system on robust pathfollowing flight for Unmanned Aerial Vehicle (UAV). Guidance and control systems on path-following flight for UAV, most based on a tracking-error-correction approach, have been studied for several years (Blajer, 1990; Baba et al., 1996, 2002; Kaminer et al., 1998; Boyle \& Chamitoff, 1999; Ochi et al., 2002; Park et al., 2004; Rysdyk, 2006). However, these systems present the difficulty of following in large tracking-error situations, e.g. steep curved path-following or tracking under wind turbulence, which might cause control saturation or divergence because the control command tends to increase as the trackingerror becomes large. One coauthor proposed a variable gain method using fuzzy logic (Baba \& Takano, 1998), which gathers and weighs on control laws according to tracking-error quantities. It performed well, but it was necessary to set some design points and to conduct several gain tunings before applying fuzzy logic.

This chapter presents a novel, simple, yet robust guidance method for path-following UAV (Sato et al., 2006; Yamasaki et al., 2007). The methodology uses pure pursuit guidance instead of traditional tracking-error correction-based methods. Pure pursuit guidance (e.g. Machol et al., 1965) demands only one gain-tuning. It produces guidance commands that are not large, with no relation to tracking error quantities. It can avoid control divergence because the pure pursuit guidance system generates guidance commands in relation to the line-of-sight angle (the angle formed by the UAV velocity vector and the line-of-sight to the target point), which is, at most, $\pi$ radian. That angle might be much less than the tracking error, e.g. $10 \mathrm{~m}$. For that reason, a robust path-following UAV can be realized assisted by pure pursuit guidance (Park et al., 2007). A target point for the UAV to orient is necessary for applying pure pursuit guidance for path-following. We introduced a receding virtual waypoint, which is placed at a proper point along a desired trajectory. This is the point of difference from the error-based reference point described in the literature (Park et al., 2007). The desired path can be provided easily in a form of a function of the trajectory arc length using cubic-spline interpolation based on some waypoints through which the UAV is presumed to fly. Once the path is generated in the form of the function of the arc length, the receding virtual waypoint, which is the target point for pure pursuit guidance, is calculable using the cubic-spline function based on the UAV flight arc length added a few seconds ahead of the future flight path length, as inferred from the UAV dynamics. This added term 
provides a future desired maneuver to the UAV, which compensates the UAV's response delay because of the UAV dynamics. Guidance commands for the UAV orientation are generated using the pure pursuit guidance with a receding virtual waypoint. The UAV can change its heading by altering the attitude. The system employs a dynamic inversion technique for the UAV's attitude control. Dynamic inversion techniques (Brockett, 1978; Lane \& Stengel, 1998; Snell et al., 1992; Baba et al., 1996), which enable the UAV to perform a nonlinear path-following ability and to maintain high maneuverability, are well-known control schemes of nonlinear dynamic systems. A dynamic inversion technique with twotime scale separation (Menon et al., 1987; Snell et al., 1992; Azam \& Singh, 1994) is applicable for UAV control, which allows the order of the dynamic inversion controller to be small. The pure pursuit guidance lets the UAV head to the target at any time. Because the pure pursuit guidance can, by its nature, head to a target point only and never works for the velocity control along the velocity vector directly, a velocity control system is necessary to maintain a desired distance from the given trajectory. The velocity controller is designed based also on the dynamic inversion approach, considering the aircraft dynamics.

First, the body axes are explained along with full, rigid body, six-degree-of-freedom, nonlinear equations of motion. Then we will discuss the guidance and control system for $\mathrm{UAV}$, which is based on the pure pursuit guidance law with a receding virtual waypoint, and the two-time scaled dynamic inversion technique, along with velocity control. Several simulations are conducted to demonstrate its path-following robustness under some random wind turbulence and steeply curved path-following situations. We also show a comparison to the tracking-error-correction-based method.

\section{Symbols and Notations}

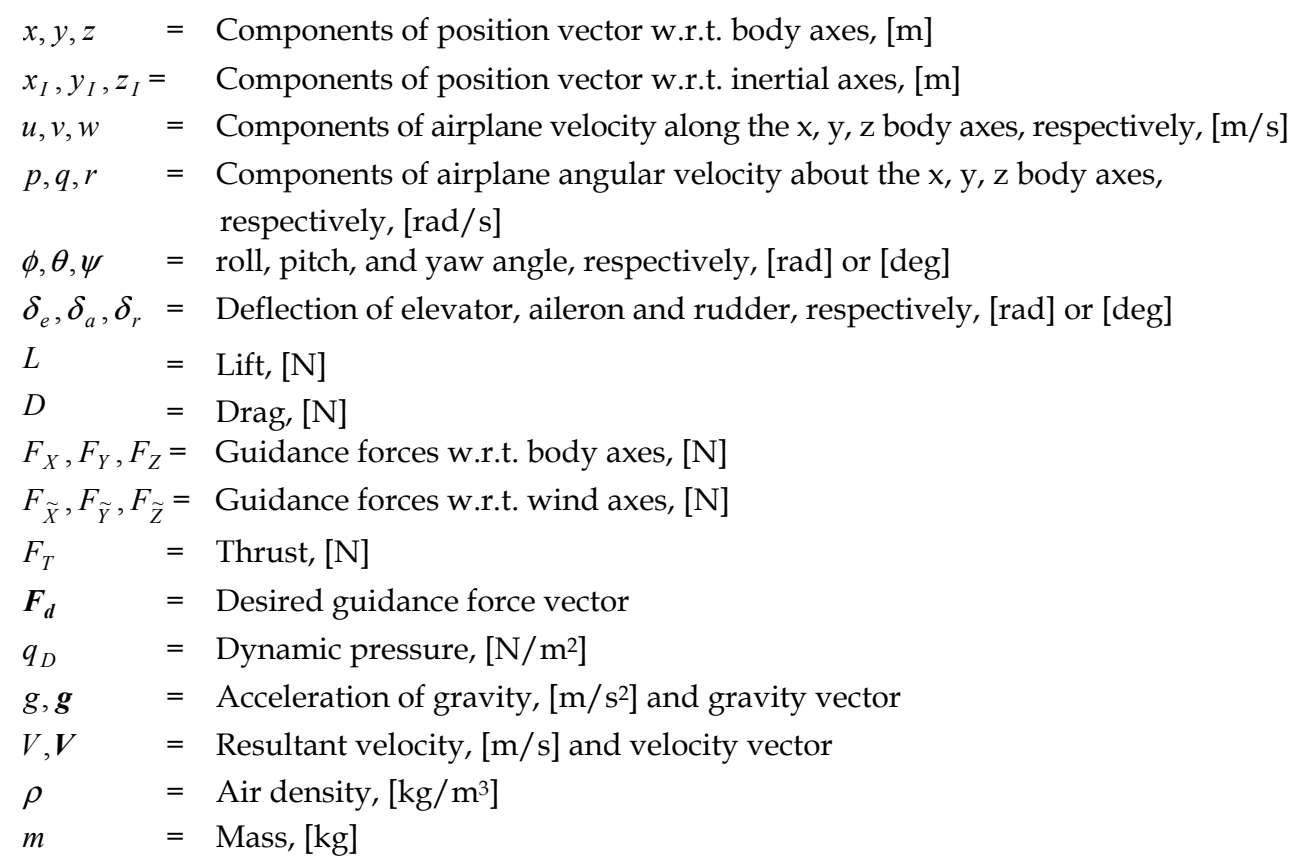




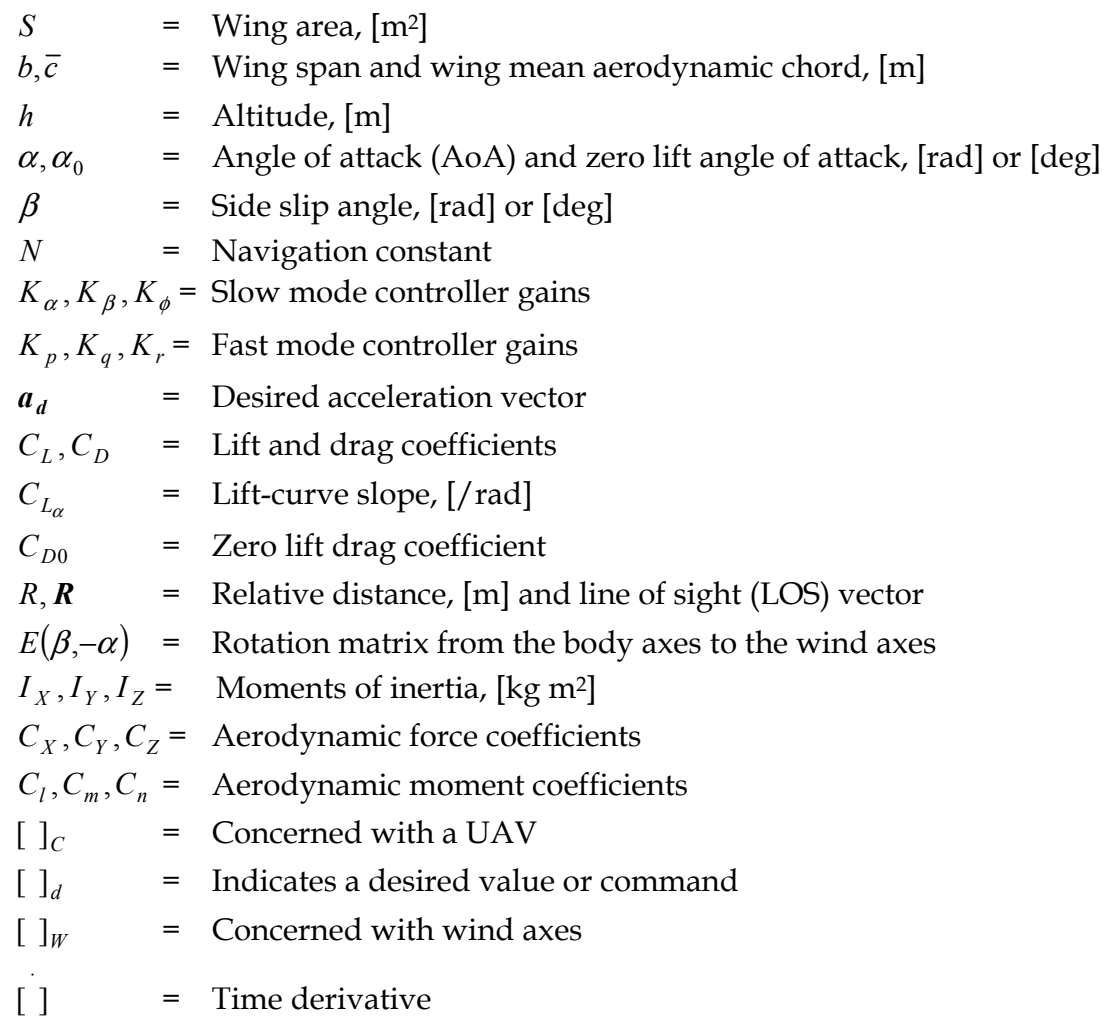

\section{Equations of motion}

Figure 1 portrays the coordinate system used for this study. The complete, six-degree-offreedom (6DOF), nonlinear, rigid-body dynamics for fixed-wing UAV with respect to the body-fixed axis system depicted in Fig. 1 are modeled by the following 12 first-order differential equations.

$$
\begin{gathered}
\dot{u}=r v-q w-g \sin \theta+\frac{q_{D} S C_{X}}{m}+\frac{F_{T}}{m} \\
\dot{v}=p w-r u+g \cos \theta \sin \phi+\frac{q_{D} S C_{Y}}{m} \\
\dot{w}=q u-p v+g \cos \theta \cos \phi+\frac{q_{D} S C_{Z}}{m} \\
\dot{p}=\frac{I_{Y}-I_{Z}}{I_{X}} q r+\frac{I_{X Z}}{I_{X}}(\dot{r}+p q)+\frac{q_{D} S b}{I_{X}} C_{l} \\
\dot{q}=\frac{I_{Z}-I_{X}}{I_{Y}} p r+\frac{I_{X Z}}{I_{Y}}\left(r^{2}-p^{2}\right)+\frac{q_{D} S \bar{c}}{I_{Y}} C_{m}
\end{gathered}
$$




$$
\begin{gathered}
\dot{r}=\frac{I_{X}-I_{Y}}{I_{Z}} p q+\frac{I_{X Z}}{I_{Z}}(\dot{p}-q r)+\frac{q_{D} S b}{I_{Z}} C_{n} \\
\dot{\phi}=p+q \sin \phi \tan \theta+r \cos \phi \tan \theta \\
\dot{\theta}=q \cos \phi-r \sin \phi \\
\dot{\psi}=q \sin \phi \sec \theta+r \cos \phi \sec \theta
\end{gathered}
$$

$\dot{x}_{I}=u \cos \theta \cos \psi+v(\sin \phi \sin \theta \cos \psi-\cos \phi \sin \psi)+w(\cos \phi \sin \theta \cos \psi+\sin \phi \sin \psi)$

$\dot{y}_{I}=u \cos \theta \sin \psi+v(\sin \phi \sin \theta \sin \psi+\cos \phi \cos \psi)+w(\cos \phi \sin \theta \sin \psi-\sin \phi \cos \psi)$

$$
\dot{z}_{I}=-u \sin \theta+v \sin \phi \cos \theta+w \cos \phi \cos \theta
$$

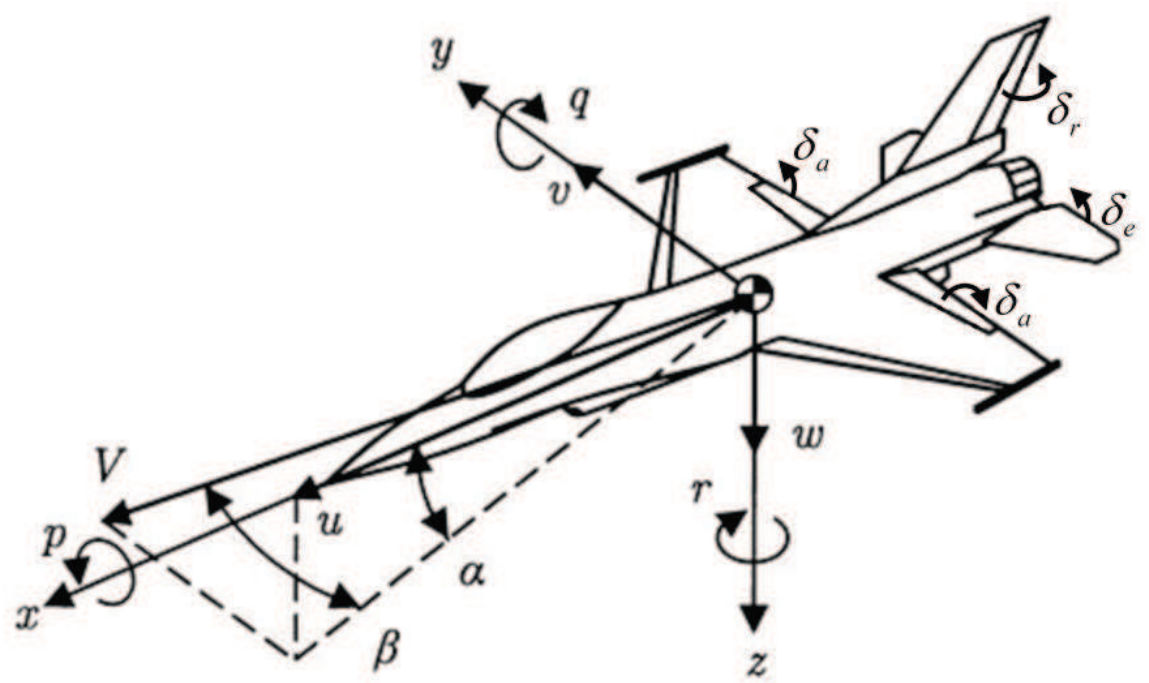

Figure 1. Coordinate system

For them, we assumed the aerodynamic coefficients appeared in the above equations as follows.

$$
\begin{gathered}
C_{X}=C_{X}\left(\alpha, \beta, \frac{\bar{c} q}{2 V}, \delta_{e}\right) \\
C_{Y}=C_{Y}\left(\alpha, \beta, \frac{b p}{2 V}, \frac{b r}{2 V}, \delta_{a}, \delta_{r}\right) \\
C_{Z}=C_{Z}\left(\alpha, \beta, \frac{\bar{c} q}{2 V}, \delta_{e}\right) \\
C_{l}=C_{l}\left(\alpha, \beta, \frac{b p}{2 V}, \frac{b r}{2 V}, \delta_{a}, \delta_{r}\right)
\end{gathered}
$$




$$
\begin{gathered}
C_{m}=C_{m}\left(\alpha, \beta, \frac{\bar{c} q}{2 V}, \delta_{e}\right) \\
C_{n}=C_{n}\left(\alpha, \beta, \frac{b p}{2 V}, \frac{b r}{2 V}, \delta_{a}, \delta_{r}\right)
\end{gathered}
$$

Equations (1-3), Eqs. (4-6), Eqs. (7-9), and Eqs. (10-12) respectively represent force equations, moment equations, rotational kinematic equations, and navigation equations. The first nine equations govern the dynamics. The navigation equations are used only for simulations to calculate the UAV position with respect to the earth frame.

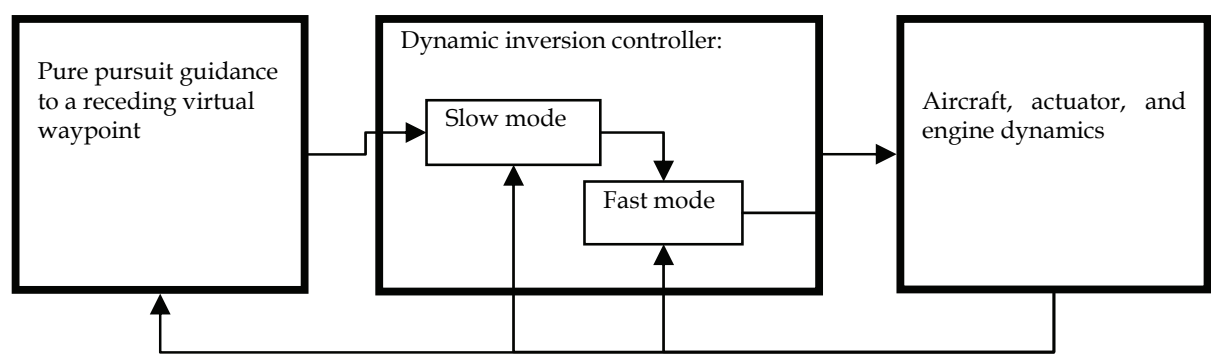

Figure 2. Guidance and control block diagram

\section{Guidance and Control System}

Figure 2 portrays a block diagram of the guidance and control system for a path-following UAV, which employs the pure pursuit guidance law for calculating the guidance command, in conjunction with a two-time-scaled dynamic inversion method (Baba et al., 1996, 2002; Sato et al., 2006; Yamasaki et al., 2007) consisting of a slow-state and a fast-state dynamic mode to generate guidance forces for nonlinear dynamic motions of the UAV. This diagram does not include the velocity control, which is discussed later.

\subsection{Receding virtual waypoints}

A desired trajectory might be used to guide a UAV along a predetermined path such as a runway approach and landing. It is useful to generate the desired trajectory from several waypoints. For this study, the cubic spline function is used because it gives the minimum curvature (Blajer, 1990; Jackson \& Crouch, 1991). Figure 3 portrays an interpolated trajectory by the cubic spline function. The circles labeled "WAYPOINT" in Fig. 3 are waypoints that are only used to generate a spline interpolated-trajectory for convenience. They differ from the receding virtual waypoint described later.

A trajectory calculated from cubic spline function can be represented using continuous functions of time or the arc length of the trajectory. The arc length is chosen as the independent variable (Baba et al., 1996, 2002; Ochi et al., 2002; Yamasaki et al., 2007), which enables the UAV to fly at various speeds. A virtual waypoint should be set as the target for the pure pursuit guidance on the generated trajectory along which the trajectory-tracking UAV are to be guided. The virtual waypoint, which is moved in each time step, is obtainable using the cubic spline function of a distance instead of using the arc length of the desired trajectory. The distance is calculated based on the UAV's flight distance added using a few- 
seconds more future flight length. Therefore, this virtual waypoint determination approach uses only the flight distance information and obviates extra information such as the tracking error components described in the literature (Park et al., 2007). If the UAV deviates from the desired trajectory, the virtual waypoint is set at a receded point in each time step according to the flight distance, including a flight distance deviated from the desired trajectory, which can avoid rapid correction control. Consequently, this virtual waypoint is designated as "a receding virtual waypoint." Pure pursuit guidance with the receding virtual waypoint can alleviate control saturation, different from the case of tracking-error-correction-based control.

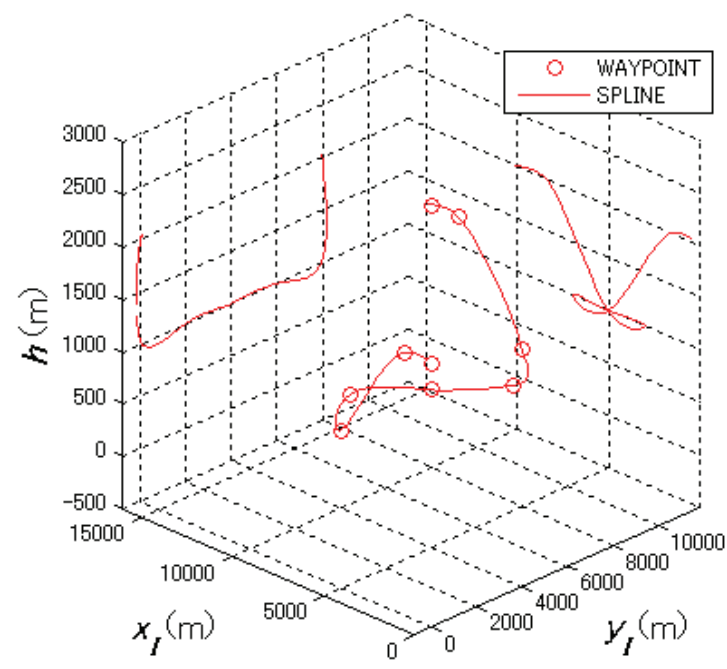

Figure 3. Interpolated trajectory using spline function of the arc length (c.f. WAYPOINT differs from the receding virtual waypoint)

\subsection{Pure pursuit guidance law}

Generally, pure pursuit guidance (PPG) is not used to guide a UAV for rendezvous or intercept purposes if the UAV velocity becomes 1.5 times greater than the target velocity because the guidance forces tend to be diverted when the relative distance between the UAV and the target becomes zero (Machol et al., 1965). However, those problems will not occur for a path-following purpose in keeping the relative distance because target points recede as the UAV moves. The relative distance can be maintained at a few degrees, not zero. Although the proportional navigation ( $\mathrm{PN})$ guidance might also be considered, the error distance from the desired trajectory is prone to remain at some few degrees because PN guidance tends to keep a line of sight (LOS) angle constant (Zarchan, 2002). In contrast, pure pursuit guidance always guides the UAV to orient in the waypoint direction in spite of both velocities. Therefore, we use the pure pursuit guidance for this study. If the target point velocity is set as zero, the characteristics of PN guidance are almost equal to that of PPG with respect to the UAV orientation direction. Assuming that the UAV pursues a receding virtual waypoint with velocity of $V_{C}$ as portrayed in Fig. 4, the following equation is 
satisfied if the line of sight (LOS) vector $\boldsymbol{R}$ and the UAV's velocity vector $\boldsymbol{V}_{\boldsymbol{C}}$ are heading in the same direction.

$$
V_{C} \times R=0
$$

To guide the UAV into the pure pursuit course, the following acceleration feedback is needed for Eq. (19).

$$
\boldsymbol{a}_{\boldsymbol{d}}=\frac{N\left(\boldsymbol{V}_{\boldsymbol{C}} \times \boldsymbol{R}\right) \times \boldsymbol{V}_{\boldsymbol{C}}}{V_{C} R}
$$

Therein, $N$ is the navigation constant. Its value is set as 2 for this study, as in a previous study (Park et al., 2007). The following equation might be preferred to using Eq. (20) for implementation purposes in the real system, especially for a large LOS angle, in applying the real LOS angle feedback.

$$
\boldsymbol{a}_{\boldsymbol{d}, \text { real }}=\frac{N\left(\boldsymbol{V}_{\boldsymbol{C}} \times \boldsymbol{R}\right) \times \boldsymbol{V}_{\boldsymbol{C}}}{V_{C} R} \frac{\sigma}{\sin \sigma}
$$

In that equation, $\sigma$ is the LOS angle. The equation means that the desired acceleration is exactly proportional to the LOS angle, as measured from, for example, a seeker's angle. However, we use Eq. (20) in this study for simplicity. From Newton's second law, the required guidance force, along with the gravity compensation, can be expressed as

$$
\boldsymbol{F}_{d}=m\left(\boldsymbol{a}_{d}-\boldsymbol{g}\right),
$$

where vector $\boldsymbol{g}$ is a gravity vector with respect to the body axes. Thereby, the desired guidance commands are obtained.

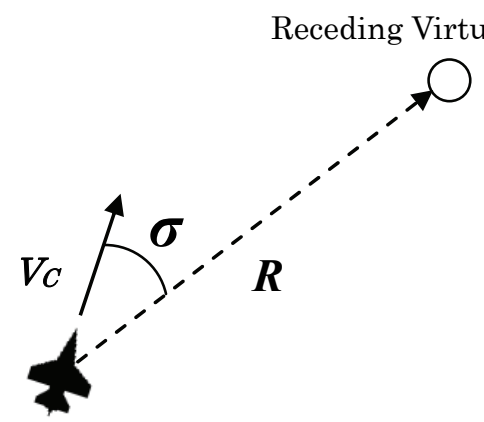

Figure 4. Position view of the UAV and the receding virtual waypoint

\subsection{Derivation of command values}

The desired guidance forces are obtained by changing the direction and magnitude of the force vector acting on the UAV. The direction and magnitude of the force vector can be changed generally by altering the UAV's slow dynamic states, i.e., angle of attack (AoA), sideslip angle, and roll (or bank) angle. The desired AoA, sideslip angle, and roll (or bank) 
angle is calculable from the desired forces in Eq. (22). The guidance forces shown in Eq. (22) related to the body axes must be transformed to wind-axis forms.

$$
\left(\begin{array}{c}
F_{\widetilde{X}} \\
F_{\widetilde{Y}} \\
F_{\widetilde{Z}}
\end{array}\right)=E(\beta,-\alpha)\left(\begin{array}{l}
F_{X} \\
F_{Y} \\
F_{Z}
\end{array}\right)
$$

In that equation, $E(\beta,-\alpha)$ represents the rotation matrix from the body axes to the wind axes. Before deriving the desired lift, $L_{d}$ and the desired roll angle, $\phi_{d}$, we assume coordinated flight, which means zero-sideslip angle. The desired AoA, sideslip angle and bank angle can be expressed by the following simple equations using guidance forces in Eq. (22) (Baba et al., 2002, Yamasaki et al., 2006, 2007).

$$
\begin{gathered}
\alpha_{d}=\frac{1}{C_{L_{\alpha}}}\left(\frac{L_{d}-F_{T} \sin \alpha}{q_{D} S}\right)+\alpha_{0} \\
\beta_{d}=0 \\
\phi_{d}=\phi+\Delta \phi_{d}
\end{gathered}
$$

In those equations, the following pertain.

$$
\begin{aligned}
& L_{d}=\sqrt{{F_{\widetilde{Y}}^{2}+F_{\widetilde{Z}}^{2}}^{2}} \\
& \Delta \phi_{d}=\tan ^{-1} \frac{F_{Y}}{-F_{Z}}
\end{aligned}
$$

The desired additional roll angle appeared in Eq. (28) is determined geometrically with respect to the body axes.

\subsection{Dynamic inversion}

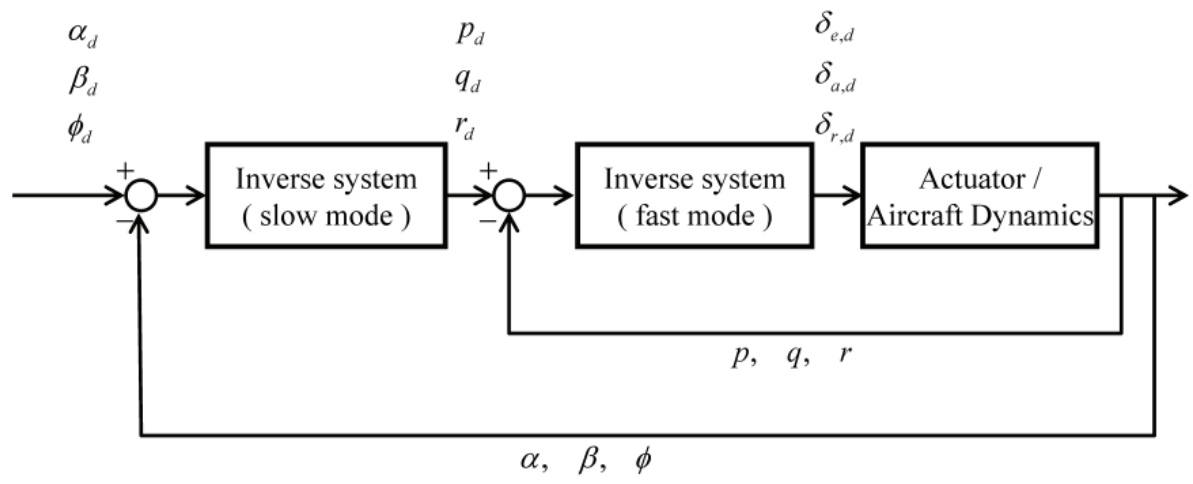

Figure 5. Block diagram of the two-time scaled dynamic inversion controller used in this study 
To maintain high maneuverability, a nonlinear dynamic controller is designed. The dynamic inversion (DI) approach is a well-known scheme for nonlinear dynamical control design. We apply for the DI approach for a conventional fixed-wing UAV. In considering control for such a UAV, attitude control is achieved by rotating the UAV. The rotation is achieved by actuating the control surfaces such as elevators, ailerons, and a rudder. The attitude control is generally conducted using two loops: rotation control loop for fast-time scale state dynamics, and attitude control loop for slow-time scale state dynamics. We used the twotime scaled DI approach (Menon et al., 1987; Snell et al., 1992; Azam \& Singh, 1994) to achieve the desired AoA, sideslip angle, and roll angle determined from the previous section. The two-time scaled DI method can reduce the controller order, which simplifies the design process. It can avoid tiresome control design problems arising in non-minimum phase systems. Figure 5 shows the schematic two-time scaled DI controller block diagrams used for this study (Baba et al., 1996; Yamasaki et al., 2006,2007). The inner loop in Fig. 5 corresponds to the fast-state dynamics: the states $p, q$, and $r$ are controlled by the rudder, elevators, and ailerons. The outer loop corresponds to the slow-state dynamics: states $\alpha, \beta$, and $\phi$, which are controlled by the body rate $p, q$, and $r$. Regarding the outer loop, it is assumed that the transient dynamics of the fast states occur so quickly that they have a negligible effect on the slow states. In the inner loop, slow-time scale variables such as $V, \alpha$, and $\beta$ are assumed to remain constant. The fast-time scale controller attempts to maintain the body rates $p_{d}, q_{d}$, and $r_{d}$ close to their values in the outer solution.

To obtain the slow state dynamics related to $V, \alpha$, and $\beta$, the following relations are used.

$$
\begin{gathered}
u=V \cos \alpha \cos \beta \\
v=V \sin \beta \\
w=V \sin \alpha \cos \beta
\end{gathered}
$$

The following are obtained after some algebraic manipulations of the above relations with Eqs. (1-3).

$$
\begin{aligned}
& \dot{V}=-\frac{q_{D} S}{m} C_{D_{W}}+\frac{F_{T}}{m} \cos \alpha \cos \beta+g(\cos \phi \cos \theta \sin \alpha \cos \beta+\sin \phi \cos \theta \sin \beta-\sin \theta \cos \alpha \cos \beta) \\
& \dot{\alpha}=-\frac{q_{D} S}{m V \cos \beta} C_{L}+q-\tan \beta(p \cos \alpha+r \sin \alpha)+\frac{g}{V \cos \beta}(\cos \phi \cos \theta \cos \alpha+\sin \theta \sin \alpha)-\frac{F_{T} \sin \alpha}{m V \cos \beta} \\
& \dot{\beta}=\frac{q_{D} S}{m V} C_{Y_{W}}+p \sin \alpha-r \cos \alpha+\frac{g}{V} \cos \beta \sin \phi \cos \theta+\frac{\sin \beta}{V}\left(g \cos \alpha \sin \theta-g \sin \alpha \cos \phi \cos \theta-\frac{F_{T} \cos \alpha}{m}\right)
\end{aligned}
$$

The last two equations and Eq. (7) form the slow-state dynamics related with $\alpha, \beta$, and $\phi$. The desired outer loop slow-state dynamics used for this study are specified by Eq. (35).

$$
\left(\begin{array}{c}
\dot{\alpha}_{d} \\
\dot{\beta}_{d} \\
\dot{\phi}_{d}
\end{array}\right)=\left(\begin{array}{c}
K_{\alpha}\left(\alpha_{d}-\alpha\right) \\
K_{\beta}\left(\beta_{d}-\beta\right) \\
K_{\phi}\left(\phi_{d}-\phi\right)
\end{array}\right) \equiv\left(\begin{array}{c}
U_{\alpha} \\
U_{\beta} \\
U_{\phi}
\end{array}\right)
$$


Therein, subscript $d$ represents the desired value. The terms $\alpha_{d}, \beta_{d}$, and $\phi_{d}$ are the commanded AoA, sideslip angle, and roll angle given by the guidance part described in Eqs. (24-26). The bandwidths $K_{\alpha}, K_{\beta}$, and $K_{\phi}$ should be slightly lower than or equal to the bandwidths of the UAV dynamics. Solving Eqs. (33-34) and (7) for $(p, q, r)^{T}$, and replacing the respective $(p, q, r)^{T}$ and $(\dot{\alpha}, \dot{\beta}, \dot{\phi})^{T}$ terms to the desired $\left(p_{d}, q_{d}, r_{d}\right)^{T}$ and $\left(U_{\alpha}, U_{\beta}, U_{\phi}\right)^{T}$ terms, one obtains the following.

$$
\left(\begin{array}{l}
p_{d} \\
q_{d} \\
r_{d}
\end{array}\right)=\left(\begin{array}{ccc}
-\cos \alpha \tan \beta & 1 & -\sin \alpha \tan \beta \\
\sin \alpha & 0 & -\cos \alpha \\
1 & \sin \phi \tan \theta & \cos \phi \tan \theta
\end{array}\right)^{-1}\left(\begin{array}{c}
U_{\alpha}-A_{\alpha} \\
U_{\beta}-A_{\beta} \\
U_{\phi}
\end{array}\right)
$$

In that equation, the following pertain.

$$
\begin{gathered}
A_{\alpha}=\left\{-q_{D} S C_{L}-F_{T} \sin \alpha+m g(\cos \phi \cos \theta \cos \alpha+\sin \theta \sin \alpha)\right\} /(m V \cos \beta) \\
A_{\beta}=\left\{q_{D} S C_{Y_{W}}-F_{T} \cos \alpha \sin \beta+(\sin \theta \cos \alpha \sin \beta+\sin \phi \cos \theta \cos \beta-\cos \phi \cos \theta \sin \alpha \sin \beta)\right\} /(m V)
\end{gathered}
$$

Equation (36) represents the inverse system of the slow-state dynamics, which yields the fast states' desired values.

The following matrix-form equation, transformed from the moment Eqs. (4-6), models the fast-state dynamics.

$$
\left(\begin{array}{c}
\dot{p} \\
\dot{q} \\
\dot{r}
\end{array}\right)=\left(\begin{array}{c}
A_{p} \\
A_{q} \\
A_{r}
\end{array}\right)+\left(\begin{array}{ccc}
B_{p a} & 0 & B_{p r} \\
0 & B_{q e} & 0 \\
B_{r a} & 0 & B_{r r}
\end{array}\right)\left(\begin{array}{l}
\delta_{a} \\
\delta_{e} \\
\delta_{r}
\end{array}\right)
$$

The following are used in that equation.

$$
\begin{gathered}
A_{p}=\left\{\begin{array}{l}
\left.X_{X Z Z}\left(I_{X}-I_{Y}+I_{Z}\right) p q+\left(-I_{Z}{ }^{2}+I_{Y} I_{Z}-I_{X Z}{ }^{2}\right) q r+I_{Z} q_{D} S b \mid C_{l_{\beta}} \beta+(b / 2 V)\left(C_{l_{p}} p+C_{l_{r}} r\right)\right] \\
\left.+I_{X Z} q_{D} S b\left[C_{n_{\beta}} \beta+(b / 2 V)\left(C_{n_{p}} p+C_{n_{r}} r\right)\right]\right\} /\left(I_{X} I_{Z}-I_{X Z}{ }^{2}\right)
\end{array}\right. \\
A_{q}=\left\{\left(I_{Z}-I_{X}\right) p r+I_{X Z}\left(r^{2}-p^{2}\right)+q_{D} S \bar{c}\left[C_{m 0}+C_{m_{\alpha}} \alpha+(\bar{c} / 2 V) C_{m_{q}} q\right]\right\} / I_{Y} \\
A_{r}=\left\{\left(I_{X Z}{ }^{2}-I_{X} I_{Y}+I_{X}{ }^{2}\right) p q-I_{X Z}\left(I_{X}-I_{Y}+I_{Z}\right) q r+I_{X Z} q_{D} S b\left[C_{l_{\beta}} \beta+(b / 2 V)\left(C_{l_{p}} p+C_{l_{r}} r\right)\right]\right. \\
\left.+I_{X} q_{D} S b\left[C_{n_{\beta}} \beta+(b / 2 V)\left(C_{n_{p}} p+C_{n_{r}} r\right)\right]\right\} /\left(I_{X} I_{Z}-I_{X Z}{ }^{2}\right) \\
B_{p a}=q_{D} S b\left(I_{Z} C_{l_{\delta_{a}}}+I_{X Z} C_{n_{\delta_{a}}}\right) /\left(I_{X} I_{Z}-I_{X Z}{ }^{2}\right) \\
B_{p r}=q_{D} S b\left(I_{Z} C_{l_{\delta_{r}}}+I_{X Z} C_{n_{\delta_{r}}}\right) /\left(I_{X} I_{Z}-I_{X Z}{ }^{2}\right) \\
B_{q e}=q_{D} S \bar{c} C_{m_{\delta_{e}}} / I_{Y}
\end{gathered}
$$




$$
\begin{aligned}
& B_{r a}=q_{D} S b\left(I_{X Z} C_{l_{\delta_{a}}}+I_{X} C_{n_{\delta_{a}}}\right) /\left(I_{X} I_{Z}-I_{X Z}{ }^{2}\right) \\
& B_{r r}=q_{D} S b\left(I_{X Z} C_{l_{\delta_{r}}}+I_{X} C_{n_{\delta_{r}}}\right) /\left(I_{X} I_{Z}-I_{X Z}{ }^{2}\right)
\end{aligned}
$$

The desired closed-loop fast-state dynamics used for this study are specified as shown below.

$$
\left(\begin{array}{c}
\dot{p}_{d} \\
\dot{q}_{d} \\
\dot{r}_{d}
\end{array}\right)=\left(\begin{array}{c}
K_{p}\left(p_{d}-p\right) \\
K_{q}\left(q_{d}-q\right) \\
K_{r}\left(r_{d}-r\right)
\end{array}\right) \equiv\left(\begin{array}{c}
U_{p} \\
U_{q} \\
U_{r}
\end{array}\right)
$$

The terms $p_{d}, q_{d}$, and $r_{d}$ are commanded, roll, pitch, and yaw rate given by the slow-state control calculated from Eq. (36). The bandwidths $K_{p}, K_{q}$, and $K_{r}$ should sufficiently exceed the bandwidths of the outer loop: $\alpha, \beta$, and $\phi$ loop, to avoid coupling between the inner and outer loop dynamics. The bandwidth corresponds to the body rate cut-off frequencies. Solving Eq. (39) for $\left(\delta_{e}, \delta_{a}, \delta_{r}\right)^{T}$ and replacing the respective $\left(\delta_{e}, \delta_{a}, \delta_{r}\right)^{T}$ and $(\dot{p}, \dot{q}, \dot{r})^{T}$ to desired $\left(\delta_{e, d}, \delta_{a, d}, \delta_{r, d}\right)^{T}$ and $\left(U_{p}, U_{q}, U_{r}\right)^{T}$, the desired control surface values are obtained as follows.

$$
\left(\begin{array}{l}
\delta_{a, d} \\
\delta_{e, d} \\
\delta_{r, d}
\end{array}\right)=\left(\begin{array}{ccc}
B_{p a} & 0 & B_{p r} \\
0 & B_{q e} & 0 \\
B_{r a} & 0 & B_{r r}
\end{array}\right)^{-1}\left(\begin{array}{l}
U_{p}-A_{p} \\
U_{q}-A_{q} \\
U_{r}-A_{r}
\end{array}\right)
$$

Equation (49) represents the inverse system of the fast states dynamics, yielding the desired control surface commands.

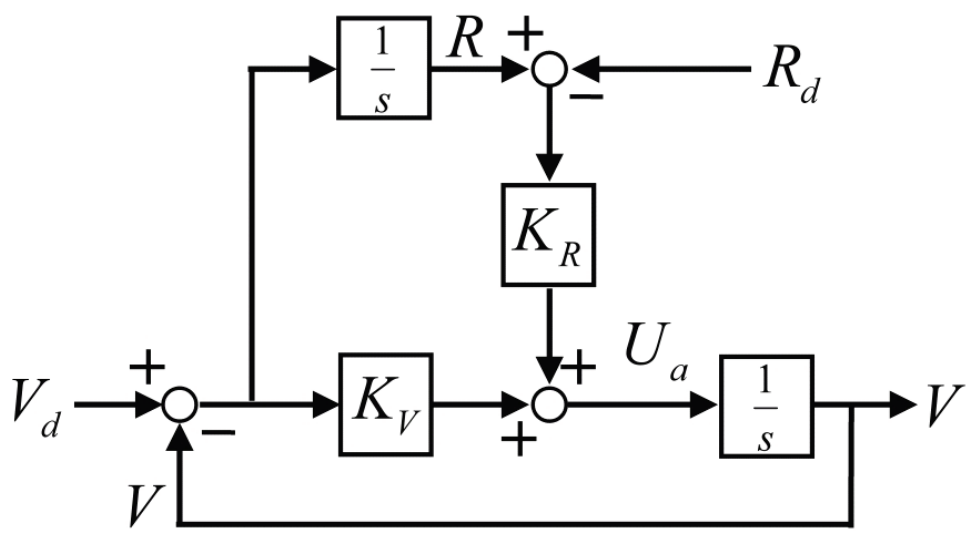

Figure 6. Block diagram of the velocity control 


\subsection{Velocity control}

The dynamic inversions described above are used only for attitude control and never work directly for the velocity control. In considering the path-following capabilities of a UAV, one must take into account its velocity control, which is necessary to maintain a desired position along the given path. Figure 6 shows a block diagram illustrating the desired velocity dynamics used for this study. The desired velocity-loop dynamics depicted in Fig. 6 are specified as

$$
\dot{\boldsymbol{V}}_{d}=K_{R}\left(R-R_{d}\right)+K_{V}\left(V_{d}-V\right) \equiv U_{a},
$$

where $K_{R}$ and $K_{V}$ respectively signify the relative distance feedback gain and the velocity feedback gain. The velocity inverse dynamics are derived from Eq. (32) by solving for the thrust input; then, replacing $\dot{V}$ by a pseudo-input $U_{a}$ as

$$
F_{T, d}=\left\{m U_{a}-m g(-\sin \theta \cos \alpha \cos \beta+\cos \theta \sin \phi \sin \beta+\cos \theta \cos \phi \sin \alpha \cos \beta)+q_{D} S C_{D_{W}}\right\} /(\cos \alpha \cos \beta)
$$

Equation (51) yields the desired thrust force commands so that the desired velocity Eq. (50) holds in the velocity dynamics of the UAV.

The UAV, as well as aircraft, has no active brake in the air. Therefore, velocity is generally controlled gradually relative to an attitude control. The gains $K_{R}$ and $K_{V}$ shown in Eq. (50) must be determined taking account of this fact. The desired velocity control system depicted in Fig. 6 is represented as shown below.

$$
\frac{V}{V_{d}}=\frac{K_{V} s+K_{R}}{s^{2}+K_{V} s+K_{R}}
$$

The characteristic equation of a general quadratic system is defined with damping ratio $\zeta$ and natural angular frequency $\omega_{n}$.

$$
s^{2}+2 \zeta \omega_{n} s+\omega_{n}^{2}=0
$$

Comparing the characteristic equation in the above equation with the desired velocity control system, the characteristics of the desired velocity dynamic system are represented as follows.

$$
\begin{gathered}
K_{R}=\omega_{n}{ }^{2} \\
K_{V}=2 \zeta \omega_{n}
\end{gathered}
$$

Because vibration of the velocity response should be alleviated for smooth path-following purpose, $\zeta$ is set around $1 ; \omega_{n}$ is determined using the following approximated equation, which is based on the approximated long-period mode for the UAV as well as general aircraft so that the velocity response converges gradually.

$$
\omega_{n} \cong \frac{\sqrt{2} g}{V}
$$


Thereby, the UAV can follow the given path with PPG with DI attitude controller and DI velocity controller. Figure 7 portrays a schematic diagram of the guidance and control system described thus far.

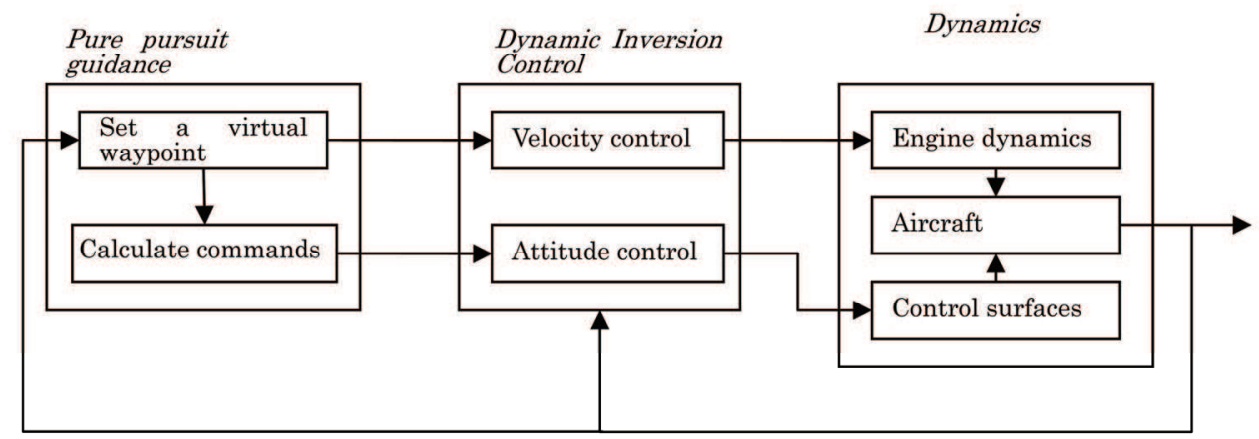

Figure 7. Schematic diagram of the guidance and control system

\section{Simulations}

These simulations use the YF-16 aircraft model (Gilbert et al., 1976) as a UAV, which includes detailed aerodynamics coefficients, the engine model, and the actuator time lags to demonstrate the guidance and control system in nonlinear 6 degree-of-freedom simulations. The following assumptions simplify the problem and demonstrate the total system.

1. The ambient atmosphere is stationary.

2. The aircraft is symmetric about the $x-z$ plane.

3. The aircraft mass is constant and the engine momentum is negligible.

4. The aircraft model is available and aerodynamic uncertainties are negligible.

5. Information of the inertial position and velocity, angular velocity, and Euler angles is available.

Assumptions 1 and 4 might be violated in some situations, but robustness for model uncertainties, external disturbances, and measurement noise might be compensated with the inner loop design of the dynamic inversion controller (Snell, 1992; Brinker \& Wise, 1996; Boyle \& Chamitoff, 1999) instead of using the proportional control appearing in Eq. (48), along with, for example, an extended Kalman filter, as appearing in the literature (Tanaka et al., 2006).

\begin{tabular}{|ll|}
\hline Parameters & Values \\
\hline Mach number & $0.6[\mathrm{Mach}]$ \\
Initial position & $(0.0,0.0,2000.0)[\mathrm{m}]$ \\
Initial heading & $0.0[\mathrm{deg}]$ \\
$R_{d}$ & $500[\mathrm{~m}]$ \\
$N$ & 2 \\
$K_{\alpha}, K_{\beta}, K_{\phi}$ & $4[\mathrm{rad} / \mathrm{s}]$ \\
$K_{p}, K_{q}, K_{r}$ & $8[\mathrm{rad} / \mathrm{s}]$ \\
Actuator time constant & $0.1[\mathrm{~s}]$ \\
\hline
\end{tabular}

Table 1. Simulation Settings 


\subsection{Simulations in stationary atmosphere}

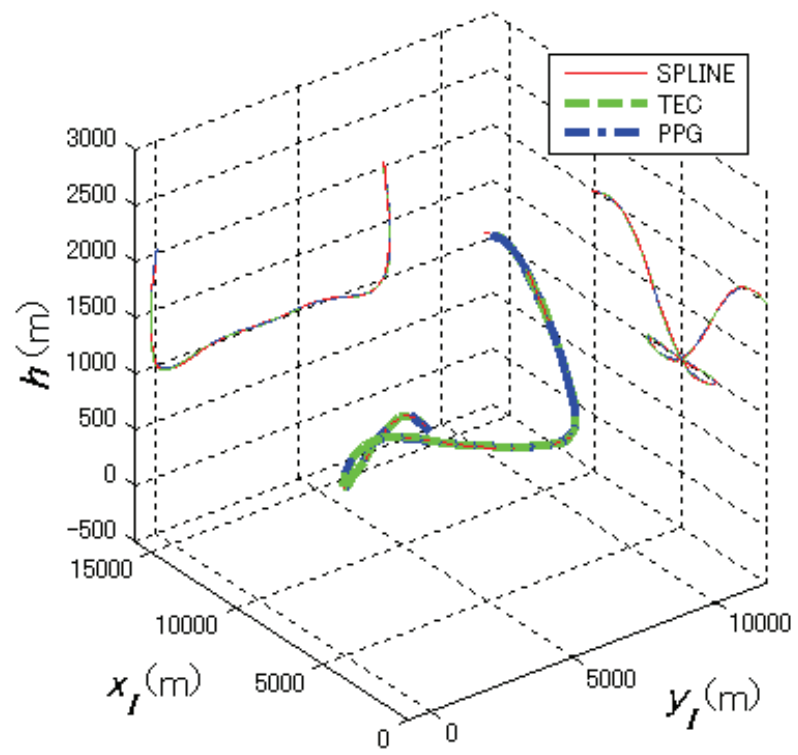

Figure 8. Tracking flight along a gentle curved path
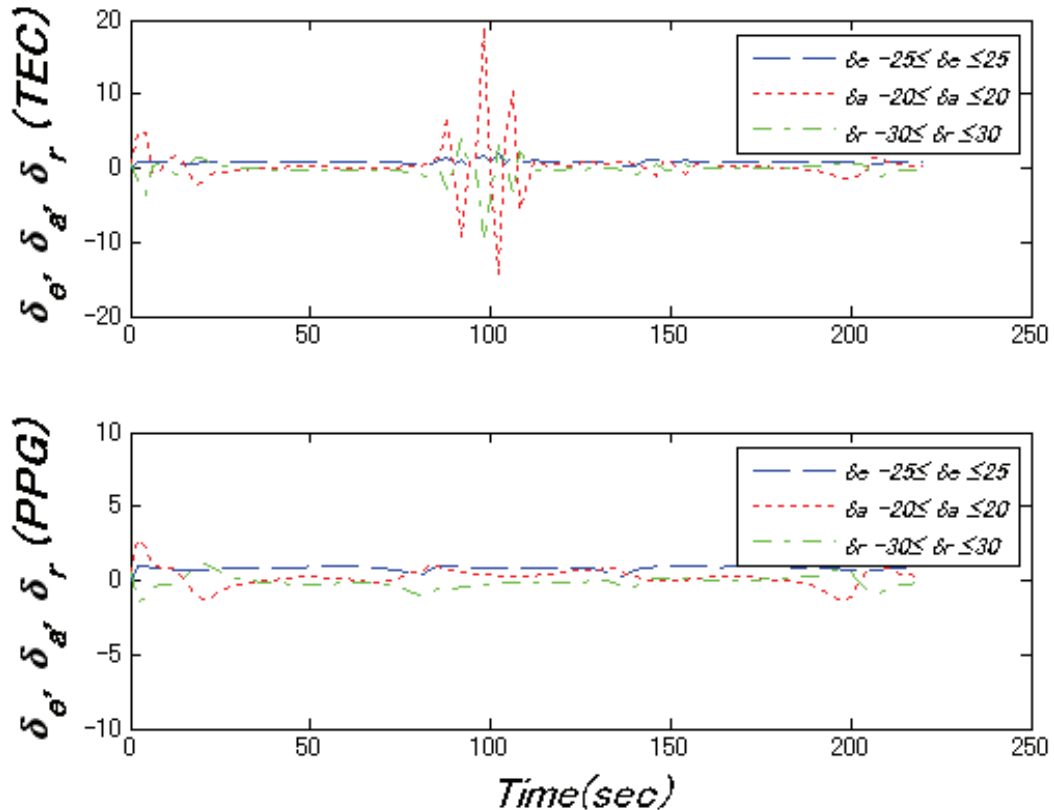

Figure 9. Control commands during path-following along the gentle curved path (deg) 


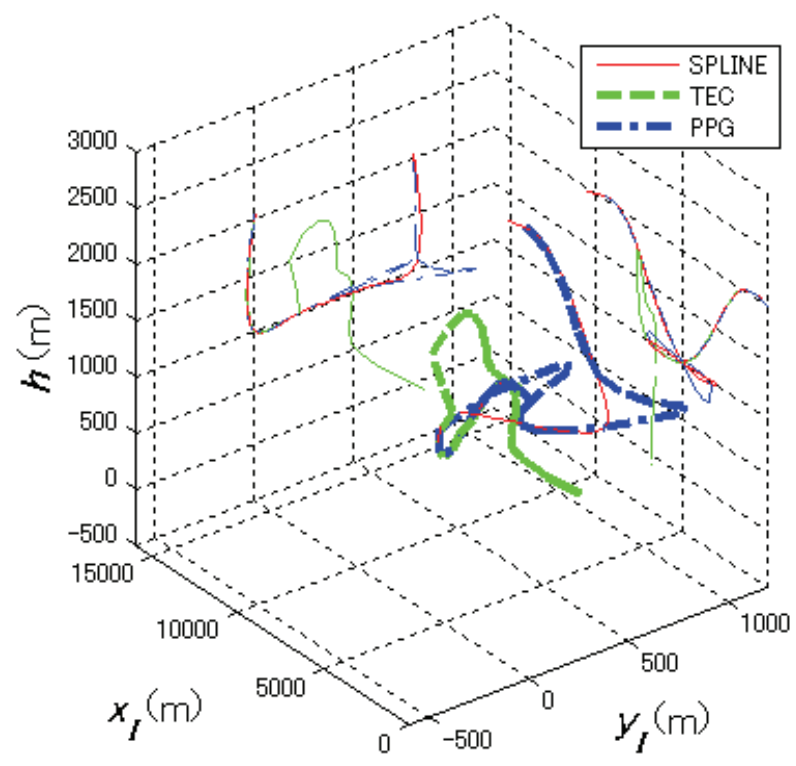

Figure 10. Tracking flight along a sharply curved path
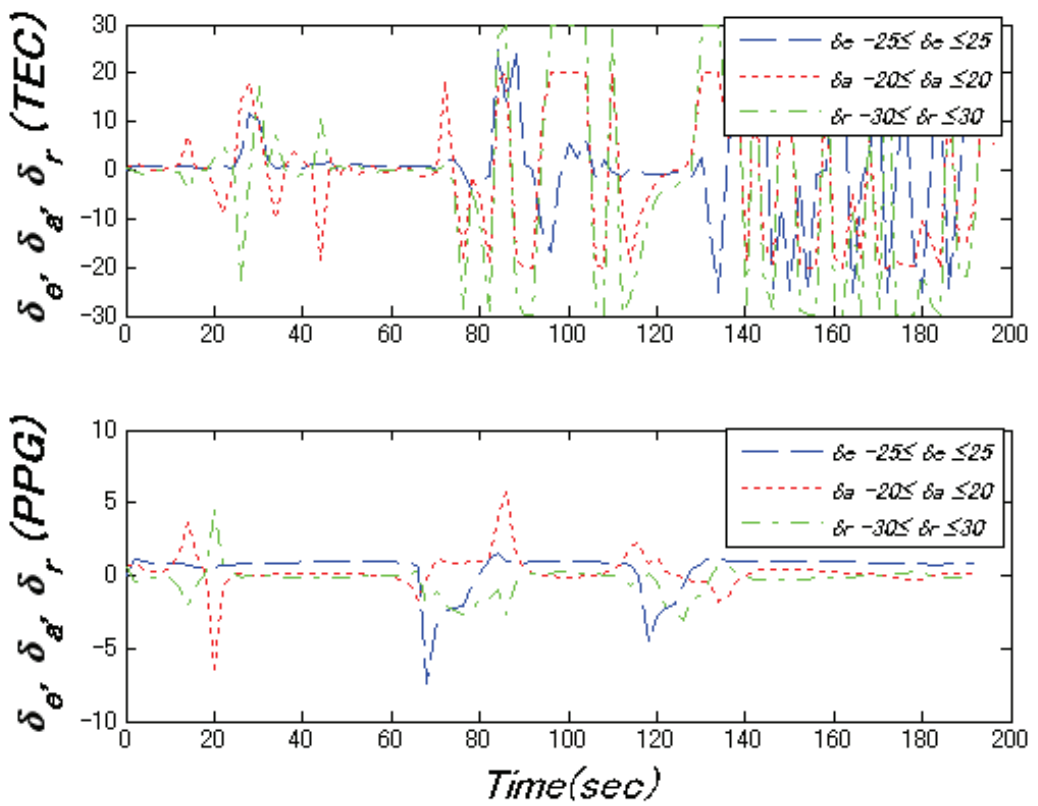

Figure 11. Control commands during path-following along the sharply curved path (deg)

Figures 8-11 portray simulation results. In Fig. 8, the solid lines labeled "SPLINE" mark the desired flight trajectories; the dashed lines labeled "TEC" and the broken dotted lines "PPG" 
respectively portray the conventional tracking-error-correction-based method (Baba, 1996) (TEC) and the pure pursuit guidance-based method with the receding virtual waypoint (PPG), along with their $(\mathrm{X}, \mathrm{h})$-plane and $(\mathrm{Y}, \mathrm{h})$-plane projections. Figure 8 shows that both TEC and the proposed PPG methods have good trajectory-tracking capability in the gentle curved trajectory. Figure 9 shows that no control saturation exists throughout the tracking flight. However, in the case of a sharply curved trajectory tracking flight, the proposed PPG method performs the high tracking ability, although the UAV deviates greatly from the desired trajectory twice whereas the conventional TEC-based method can not follow the trajectory when the UAV is deviated from the trajectory (Fig. 10). Figure 11 shows that the TEC-based method causes control saturation, although the proposed PPG method does not.

\subsection{Simulations in wind turbulence circumstance}

The Dryden wind turbulence model (Parris, 1975) is used for wind perturbation generation. A wind shear model is added to the wind turbulence for setting some averaged wind speed. Turbulence scales and its intensities are determined from prior specifications (MIL-F-8785C, 1980) assisted by linear interpolation for the lack of information range. The wind shear model presented in the specifications (MIL-F-8785C, 1980) is used in the simulations for averaged wind speed generation. As in the previous section, two simulations are conducted: a gentle curved path following and a sharply curved path case. Table 2 shows the turbulence model simulation settings. Other settings are as in Table 1.

\begin{tabular}{|ll|}
\hline Parameters & Values \\
\hline Reference altitude for turbulence scales & $1000[\mathrm{~m}]$ \\
Turbulence intensity & Moderate \\
Initial heading & $0.0[\mathrm{deg}]$ \\
Averaged wind at $6.1[\mathrm{~m}]$ & $6.528[\mathrm{~m} / \mathrm{s}]$ \\
Averaged wind direction & East to West \\
& (Horizontal blow) \\
\hline
\end{tabular}

Table 2. Simulation Settings
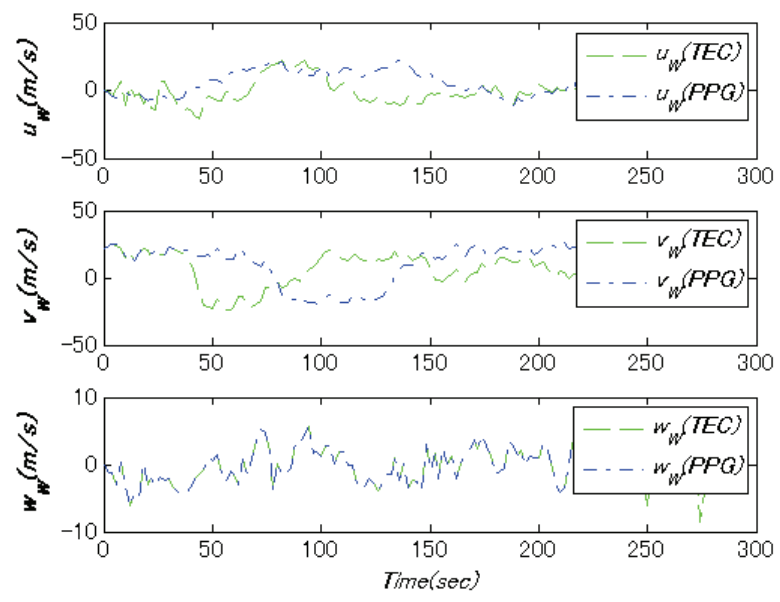

Figure 12. Time histories of wind turbulence with respect to the body axis components 
Figure 12 presents an example of generated wind turbulence components related to the body axes, which are exerted to the UAV guided by the TEC method and the proposed PPG method. That figure shows that the wind turbulence has non-zero averaged velocity. The wind velocity components are frozen to the earth frame: the wind velocities in each simulation are equal when the UAVs fly through the same path, but each component is varied on every field, thereby producing the different velocity components and different result in each simulation.

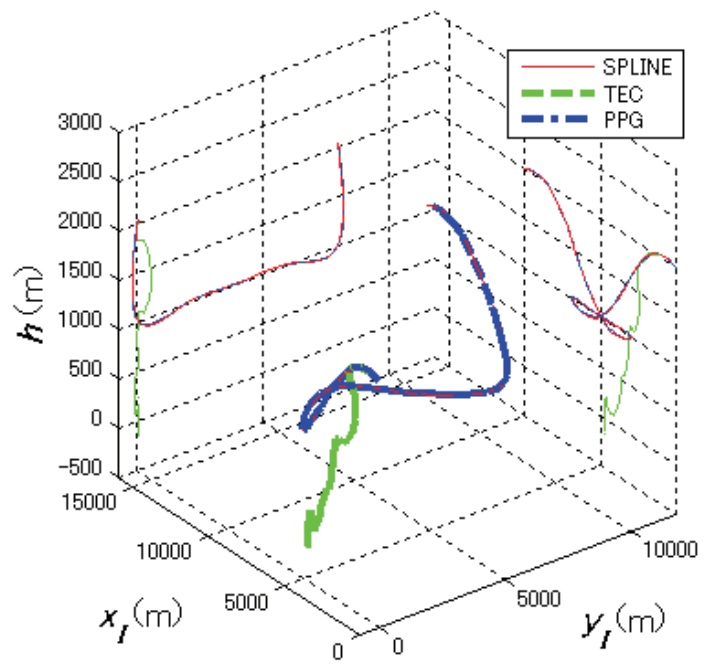

Figure 13. Tracking flight along a gentle curved path under wind turbulence
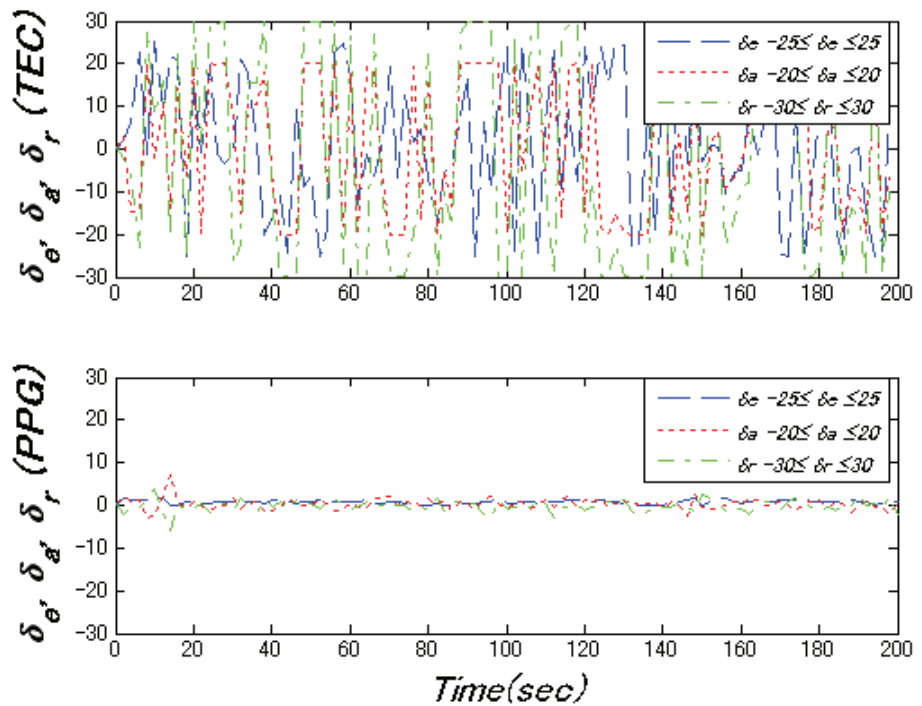

Figure 14. Control commands during path-following along the gentle curved path under wind turbulence (deg) 
Figures 13-16 show simulation results under wind turbulence. The conventional TEC-based guidance method fails to follow the desired trajectory, even in gently curved trajectory in wind turbulence, whereas the proposed PPG method can recover from the deviation and finally follow the trajectory, even in the sharply curved path-following case. These figures show that the PPG method with the reseeding waypoint has robustness relative to external wind turbulence and to deviation from the desired trajectory, whereas the conventional TEC-based guidance method does not.

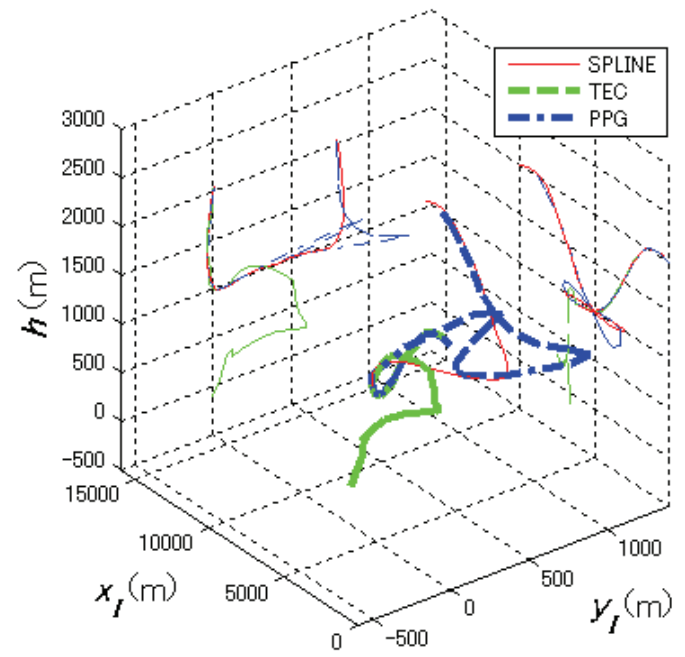

Figure 15. Tracking flight along a sharply curved path under wind turbulence
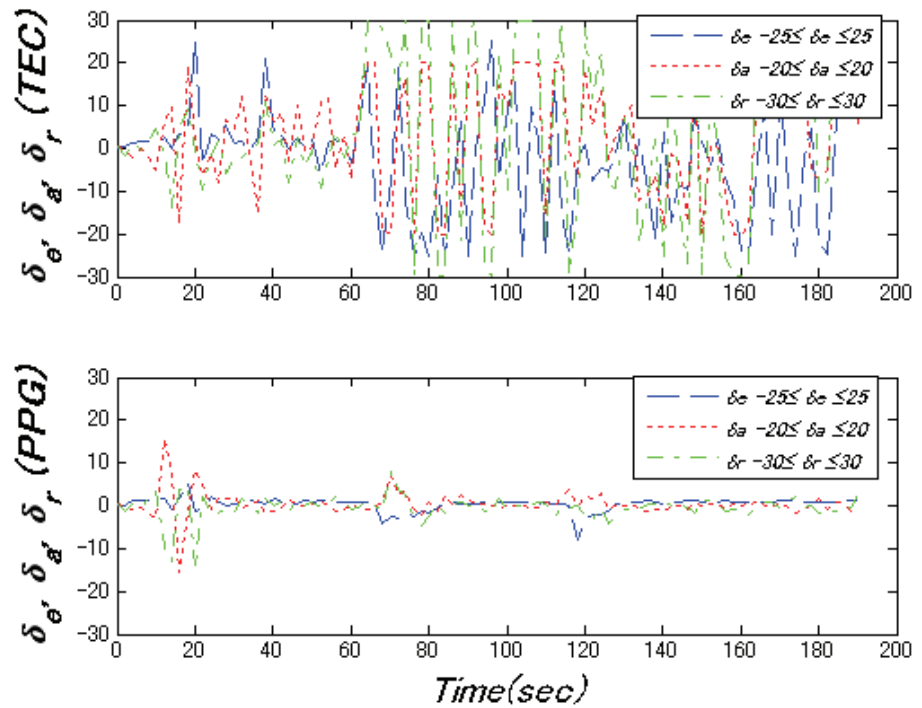

Figure 16. Control commands during path-following along the sharply curved path under wind turbulence (deg) 


\section{Conclusions}

A robust path-following guidance and control system for a UAV is introduced. Pure pursuit guidance with a receding virtual waypoint is proposed to achieve robust path-following capability for the UAV. The system enables the UAV to recover from huge-tracking-error situations alleviating control saturation. The UAV can be equipped with robustness for any sharply curved path-following and wind turbulence circumstances. An optimal navigation constant according to a given path and a proper receding virtual waypoint position considering a UAV dynamics should be analyzed in the future.

\section{References}

Azam, M. and Singh, S. N. (1994). Invertibility and Trajectory Control for Nonlinear Maneuvers of Aircraft, Journal of Guidance, Control, and Dynamics AIAA, Vol. 17, No. 1, pp. 192-200

Baba, Y., Takano, H. and Sano, M. (1996). Desired Trajectory and Guidance Force Generators for an Aircraft, Proc. Guidance, Navigation and Control Conference AIAA, 96-3873

Baba, Y. and Takano, H. (1998). Robust Flight Trajectory Tracking Control Using Fuzzy Logic, Proc. the 8th ISDGEA, Maastricht, pp. 68-75

Baba, Y., Takano, H., Miyamoto, S. and Ono, K. (2002). Air Combat Guidance Law for an UCAV, Proc. 1st UAV Conference, AIAA-2002-3427, Virginia

Blajer, W. (1990). Aircraft program motion along a predetermined trajectory. Part II. Numerical simulation with application of spline functions to trajectory definitions, Aeronautical Journal, Vol. 94, pp. 53-58

Boyle, D. P. and Chamitoff, G. E. (1999). Autonomous Maneuver Tracking for Self-Piloted Vehicles, Journal of Guidance, Control, and Dynamics, Vol. 22, No. 1, AIAA, pp. 58-67

Brinker, J. S. and Wise, K. A. (1996). Stability and Flying Qualities Robustness of a Dynamic Inversion Aircraft Control Law, Journal of Guidance, Control, and Dynamics, Vol. 19, No. 6, pp. 1270-1277

Brockett, R. W. (1978). Feedback Invariants for Nonlinear Systems, Proc. the 1978 IFAC World Congress, pp. 1115-1120

Gilbert, W. P., Nguyen, L. T. and Van Gunst, R. W. (1976). Simulator Study of the Effectiveness of an Automatic Control System Designed to Improve the HighAngle-of-Attack Characteristics of a Fighter Airplane, TN D-8176, NASA

Jackson, J. W. and Crouch, P. E. (1991). Dynamic Interpolation and Application to Flight Control, Journal of Guidance, Control and Dynamics, Vol. 14, No. 4, pp. 814-822

Kaminer, I., Pascoal, A., Hallberg, E. and Silvestre, C. (1998). Trajectory Tracking for Autonomous Vehicles: An Integrated Approach to Guidance and Control, Journal of Guidance, Control and Dynamics, Vol. 21, No. 1, 29-38

Lane, S. H. and Stengel, R. F. (1988). Flight Control Design Using Non-linear Inverse Dynamic, Automatica, Vol. 24, pp. 471-483

Machol, R. E., Tanner, Jr., W. P. and S. N. Alexander (1965). System Engineering Handbook, Chap. 19 Guidance, R. E. Howe, McGraw-Hill Book Company

Military Specification, U. S. (1980). MIL-F-8785C, 5th November

Menon, P. K. A., Badgett, M. E., Walker, R. A. and Duke, E. L. (1987). Nonlinear Flight Test Trajectory Controller for Aircraft, Journal of Guidance, Vol. 10, No. 1, AIAA, pp. 6772 
Ochi, S., Takano, H. and Baba, Y. (2002). Flight Trajectory Tracking System applied to inverse control for aerobatic maneuvers, Inverse Problems in Engineering Mechanics III, Elsevier Science Ltd., pp. 337-344

Park, S., Deyst, J. and How, J. P. (2004). A New Nonlinear Guidance Logic for Trajectory Tracking, Proc. Guidance, Navigation and Control Conference AIAA 2004-4900

Park, S., Deyst, J. and How, J. P. (2007). Performance and Lyapunov Stability of a Nonlinear Path-Following Guidance Method, Journal of Guidance, Control, and Dynamics AIAA, Vol. 30, No. 6, pp. 1718-1728

Parris, B. L. (1975). Modeling Turbulence for Flight Simulations at NASA-AMES, CSCR No. 4, NASA

Rysdyk, R. (2006). Unmanned Aerial Vehicle Path Following for Target Observation in Wind, Journal of Guidance, Control, and Dynamics, Vol. 29, No. 5, pp. 1092-1100

Sato, Y., Yamasaki, T., Takano, H. and Baba, Y. (2006). Trajectory Guidance and Control for a Small UAV, KSAS International Journal, Vol. 7, No. 2, pp. 137-144

Snell, S. A. (1992). Preliminary Assessment of the Robustness of Dynamic Inversion Based Flight Control Laws, AIAA-92-4330-CP, pp. 206-216

Snell, S. A., Enns, D. F. and Garrard Jr., W. L. (1992). Nonlinear Inversion Flight Control for a Supermaneuverable Aircraft, Journal of Guidance, Control, and Dynamics AIAA, Vol. 15, No. 4, pp. 976-984

Tanaka, N., Suzuki, S., Masui, K. and Tomita, H. (2006). Restructurable Guidance and Control for Aircraft with Failures Considering Gust Effects, Journal of Guidance, Control, and Dynamics, Vol. 29, No. 3, AIAA, pp. 671-679

Yamasaki, T., Enomoto, K., Tanaka, D., Takano, H. and Baba, Y. (2006). Automatic Control for Chase Aircraft, KSAS International Journal, Vol. 7, No. 2, pp. 145-154

Yamasaki, T., Sakaida, H., Enomoto, K., Takano, H. and Baba, Y. (2007). Robust TrajectoryTracking Method for UAV Guidance Using Proportional Navigation, Proc. International Conference on Control, Automation and Systems, Seoul, pp. 1404-1409

Zarchan, P. (2002). Tactical and Strategic Missile Guidance - Fourth Edition - Progress in Astronautics and Aeronautics AIAA, Vol. 176, ISBN-13: 978-1-56347-874-1, Virginia 


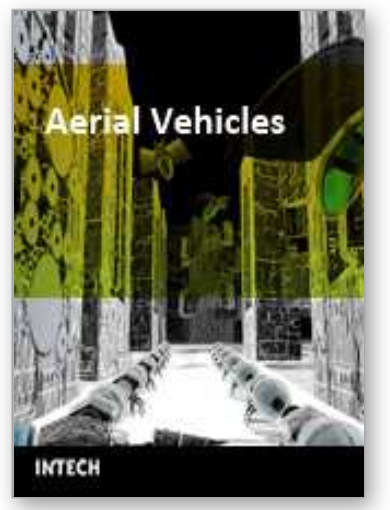

\author{
Aerial Vehicles \\ Edited by Thanh Mung Lam
}

ISBN 978-953-7619-41-1

Hard cover, 320 pages

Publisher InTech

Published online 01, January, 2009

Published in print edition January, 2009

This book contains 35 chapters written by experts in developing techniques for making aerial vehicles more intelligent, more reliable, more flexible in use, and safer in operation. It will also serve as an inspiration for further improvement of the design and application of aeral vehicles. The advanced techniques and research described here may also be applicable to other high-tech areas such as robotics, avionics, vetronics, and space.

\title{
How to reference
}

In order to correctly reference this scholarly work, feel free to copy and paste the following:

Takeshi Yamasaki, Hiroyuki Takano and Yoriaki Baba (2009). Robust Path-Following for UAV Using Pure Pursuit Guidance, Aerial Vehicles, Thanh Mung Lam (Ed.), ISBN: 978-953-7619-41-1, InTech, Available from: http://www.intechopen.com/books/aerial_vehicles/robust_path-

following_for_uav_using_pure_pursuit_guidance

\section{INTECH}

open science | open minds

\section{InTech Europe}

University Campus STeP Ri

Slavka Krautzeka 83/A

51000 Rijeka, Croatia

Phone: +385 (51) 770447

Fax: +385 (51) 686166

www.intechopen.com

\section{InTech China}

Unit 405, Office Block, Hotel Equatorial Shanghai

No.65, Yan An Road (West), Shanghai, 200040, China

中国上海市延安西路65号上海国际贵都大饭店办公楼405单元

Phone: $+86-21-62489820$

Fax: $+86-21-62489821$ 
(C) 2009 The Author(s). Licensee IntechOpen. This chapter is distributed under the terms of the Creative Commons Attribution-NonCommercialShareAlike-3.0 License, which permits use, distribution and reproduction for non-commercial purposes, provided the original is properly cited and derivative works building on this content are distributed under the same license. 